Ergod. Th. \& Dynam. Sys. (1986), 6, 489-503

Printed in Great Britain

\title{
Dynamics of entire functions near the essential singularity
}

\author{
ROBERT L. DEVANEY AND FOLKERT TANGERMAN \\ Department of Mathematics, Boston University, Boston, Mass. 02215, USA
}

(Received 4 December 1985)

\begin{abstract}
We show that entire functions which are critically finite and which meet certain growth conditions admit 'Cantor bouquets' in their Julia sets. These are invariant subsets of the Julia set which are homeomorphic to the product of a Cantor set and the line $[0, \infty)$. All of the curves in the bouquet tend to $\infty$ in the same direction, and the map behaves like the shift automorphism on the Cantor set. Hence the dynamics near $\infty$ for these types of maps may be analyzed completely. Among the entire maps to which our methods apply are exp $(z), \sin (z)$, and $\cos (z)$.
\end{abstract}

\section{Introduction}

In recent years there has been an explosion of interest in the dynamics of complex analytic maps. The important works of Mandelbrot [Ma], Douady-Hubbard (DH1], and Sullivan [S], among many others, have fuelled this interest. Most of this work has dealt with the dyanmics of either polynomials or rational maps, but recently, there has been increasing interest in the behaviour of entire, transcendental functions ([DK], [BR], [GK], [EL2]).

The goal of this paper is to describe the structure of the Julia set of a large class of entire functions. The Julia set of a map $E$, denoted by $J(E)$, is the set which carries all of the complicated or chaotic dynamics of the map. It may be defined as the set of points at which the family of iterates of the map, $E, E^{2}=E \circ E, E^{3}, \ldots$, fails to be a normal family of functions. Equivalently, $J(E)$ is the closure of the set of repelling periodic points of the map, as was proved by Fatou [F] and Baker [Ba]. See [B] for background on the Julia sets of complex analytic maps.

The Julia set of an entire transcendental function is necessarily quite different from that of a polynomial or a rational map. For one thing, the Julia set of an entire transcendental function is never compact, as it is for polynomials and rational maps. In addition, the essential singularity at $\infty$ injects a huge amount of hyperbolicity or stretching into the map. Indeed, by the Great Picard theorem, one iteration of the map on any neighbourhood of $\infty$ covers the entire plane, missing at most one point. This type of hyperbolicity serves to increase the complexity of the dynamics on the Julia set.

In this paper we will show that the geometry of the Julia sets of entire maps is quite different from that of polynomials. For a large class of entire maps, we will show that the Julia set consists of a collection of what we call 'Cantor $N$-bouquets'. 
Roughly speaking, a Cantor bouquet is the Cartesian product of a Cantor set $C_{N}$ with the closed half-line $[0, \infty)$. On this set, the map behaves in a simple manner, dynamically speaking. On $C_{N} \times\{0\}, E$ reduces to the well-understood shift automorphism. $E$ simply permutes the 'tails' or 'hairs' $c \times[0, \infty)$ according to this shift automorphism. On these tails, all points tend to infinity under iteration of $E$, with the exception of $c \times\{0\}$, whose orbit remains bounded.

Thus the Julia set for our class of maps may be visualized as containing a collection of Cantor sets of curves, and the map on each of these sets may be readily understood.

The class of maps to which our results apply are those entire functions which are crtitically finite (i.e. only finitely many critical and asymptotic values) and which meet certain growth conditions. These conditions are described in detail in $\S 3$, where we introduce the notion of a hyperbolic exponential tract with asymptotic direction. In $\S 1$ we discuss the basic properties of Cantor bouquets, and in $\S 2$ we show how Cantor bouquets arise in a specific example, the complex exponential map. Later, in $\$ 4$, we show that our methods apply to a variety of important entire functions, including $\sin z$ and $\cos z$.

It is a pleasure to acknowledge helpful conversations with Lisa Goldberg and Linda Keen while this paper was being written.

\section{Cantor bouquets}

Let $E$ be an entire transcendental function which is critically finite, i.e. which has finitely many critical and asymptotic values. This class of entire functions includes some of the most important transcendental functions such as $e^{z}, \sin (z)$, and $\cos (z)$. They are also among the simplest entire functions dynamically; for example, as was recently shown by Goldberg and Keen [GK] and Eremenko and Ljubic [EL1], they do not possess wandering domains. It is known that wandering domains may exist for certain entire, transcendental functions [Ba2], [Ba3], although they do not exist for rational maps [S].

Let $D$ be an open disk in the plane which contains all of the critical and (finite) asymptotic values. Let $\Gamma$ be the complement of $D$.

THEOREM 1.1 (Existence of exponential tracts). Let $E$ be an entire, transcendental function which is critically finite.

(1) Any connected component $T$ of $E^{-1}(\Gamma)$ is a disk whose closure contains $\infty$.

(2) $E: T \rightarrow \Gamma$ is a universal covering.

Proof. We first show that $E: E^{-1}(\Gamma) \rightarrow \Gamma$ is a covering map. Let $z \in$ int $\Gamma$ and let $B$ be an open disk about $z$ in $\Gamma$. Let $U$ be a component of $E^{-1}(B)$. If $\bar{U}$ is compact, then $E: U \rightarrow B$ is a diffeomorphism, since $E^{\prime} \neq 0$ in $\bar{U}$. If $\bar{U}$ is non-compact, let $q \in B$ and choose an exhaustion of $\bar{B}$ by simple closed curves $\gamma_{t}$ for $0 \leq t \leq 1$ with $\gamma_{0}=\{q\} . E^{-1}\left(\gamma_{t}\right)$ is a simple closed curve for $t$ small. Let $s$ be the smallest real for which $E^{-1}\left(\gamma_{s}\right)$ is not a simple closed curve. $E^{-1}\left(\gamma_{s}\right)$ is a submanifold of $C$, since $E^{\prime} \neq 0$ on $\bar{U}$. Hence $E^{-1}\left(\gamma_{s}\right)$ extends to infinity. Thus we may choose a curve in $E^{-1}(\bar{B})$ tending to $\infty$ which is mapped into $\gamma_{s}$. This yields an asymptotic value in $\bar{B}$. Hence $E \mid E^{-1}(\Gamma)$ is a covering map. 
Now $\Gamma$ is a punctured disk in $C \cup\{\infty\}$. Hence any component $T$ is either a punctured disk or a disk. In the latter case $E \mid T$ is a universal covering, as required. In the former case, if the puncture is at $\infty, E$ is a polynomial, contrary to our assumption that $E$ is transcendental. If the puncture is at $a \neq \infty$, then $E$ has a pole at $a$, which contradicts the fact that $E$ is entire. This completes the proof.

We call a component $T$ of $E^{-1}(\Gamma)$ an exponential tract. In such a region, we may write $E(z)=\exp (\phi(z))$ for $z \in T$ and some analytic map $\phi$.

Our goal is to understand the behaviour of $E$ on the Julia set near infinity. Points which leave $E^{-1}(\Gamma)$ under iteration fall into $D$ and hence leave a neighbourhood of $\infty$. We will exclude these points from consideration. Given an exponential tract $T$, let $J_{T}(E)=\left\{z \in J(E) \mid E^{n}(z) \in T\right.$ for all $\left.n\right\}$. Clearly, $J_{T}(E)$ is a closed, invariant subset of $J(E)$. Our goal is to analyze the topological structure of $J_{T}(E)$ as well as the dynamics of $E$ on this set.

As in other types of dynamical systems, Cantor sets and shift automorphisms often arise as invariant sets for entire maps. Let $N \in \mathbb{Z}^{+}$and define

$$
\Sigma_{N}=\left\{(s)=\left(s_{0} s_{1} s_{2} \ldots\right)\left|s_{j} \in \mathbb{Z},\right| s_{j} \mid \leq N\right\} \text {. }
$$

$\Sigma_{N}$ consists of all infinite sequences of integers less than or equal to $N$ in absolute value. It is well known that, with the product topology, $\Sigma_{N}$ is homeomorphic to a Cantor set.

Define $\sigma: \Sigma_{N} \rightarrow \Sigma_{N}$ by $\sigma\left(s_{0} s_{1} s_{2} \ldots\right)=\left(s_{1} s_{2} s_{3} \ldots\right)$; i.e. $\sigma$ 'forgets' the first entry of a sequence. The map $\sigma$ is known as the shift automorphism. The following properties of $\sigma$ are well known (see [Sm]).

Proposition 1.2. (i) Periodic points of $\sigma$ are dense in $\Sigma_{N}$.

(ii) $\sigma$ has a dense orbit, i.e. $\sigma$ is topologically transitive.

We call an invariant subset $C$ of $J(E)$ an $N$-bouquet for $E$ if

(1) There is a homeomorphism $h: \Sigma_{N} \times[0, \infty) \rightarrow C$.

(2) $\pi \circ h^{-1} \circ E \circ h(s, t)=\sigma(s)$, where $\pi: \Sigma_{N} \times[0, \infty) \rightarrow \Sigma_{N}$ is the projection map.

(3) $\lim _{t \rightarrow \infty} h(s, t)=\infty$.

(4) $\lim _{n \rightarrow \infty} E^{n} \circ h(s, t)=\infty$ if $t \neq 0$.

An $N$-bouquet includes naturally in an $(N+1)$-bouquet by considering only sequences with entries less than or equal to $N$ in absolute value.

The invariance of $C$ requires that $E(h(s, 0))=h(\sigma(s), 0)$. Hence the set of points $\Lambda=h(s, 0)$ is an invariant set on which $E$ is topologically conjugate to the shift. We call $\Lambda$ the crown of $C$. The curve $h_{s}(t)$ for $t>0$ is called the tail associated to $s$. We view each tail as comprising a piece of the 'stable manifold' of infinity.

The following situation often arises for entire maps. Let $C_{n}$ be an $n$-bouquet and suppose $C_{n} \subset C_{n+1} \subset \cdots$ is an increasing sequence of bouquets with the natural inclusion maps. The set

is then called a Cantor bouquet.

$$
C=\overline{\bigcup_{n \geq 0} C_{n}}
$$

In the next section we will work out an explicit example of a Cantor bouquet which occurs for the complex exponential family. 


\section{The exponential map}

Cantor bouquets arise very often in the dynamics of entire maps which are critically finite, as we will show in $\$ 3$. Here we describe in some detail the simplest example of a Cantor bouquet. In the last section, we will extend these ideas to other important examples.

Let $E_{\lambda}=\lambda \exp (z)$, where $0<\lambda<1 / e$. The graph $E_{\lambda}$ on $\mathbb{R}$ is depicted in figure 1 .

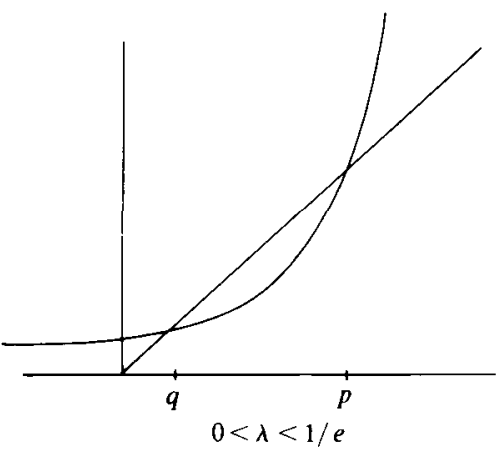

Figure 1

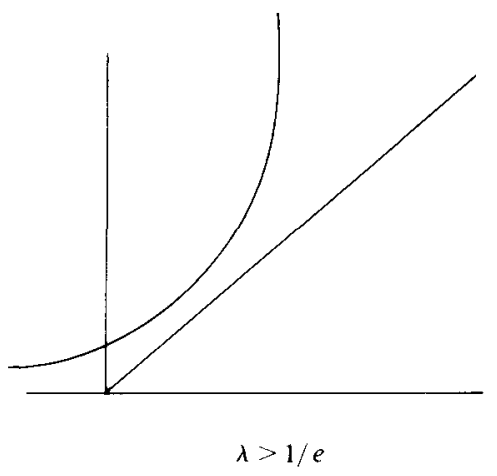

$\lambda>1 / e$

Note that $E_{\lambda}$ has two fixed points, an attracting fixed point at $q<1$ and a repelling fixed point at $p>1$. Since $E_{\lambda}$ maps the vertical line $x=p$ onto the circle of radius $p$, it follows that all points with $\operatorname{Re} z<p$ lie in the basic of attraction of $q$. Hence, $J\left(E_{\lambda}\right)$ lies to the right of this line. More can be said:

Proposition 2.1. The basin of attraction of $q$ is open and dense in $C . J(E)$ is the complement of this basin.

Proof. Suppose $D$ is an open disk in the complement of the basin of $q$. Hence $\operatorname{Re}\left(E_{\lambda}^{n}(z)\right)>1$ for all $n$ and all $z \in D$. But then $\left|\left(E_{\lambda}^{n}\right)^{\prime}(z)\right|>1$, so $E_{\lambda}^{n}$ expands $D$ for each $n$. In particular, there exists $n$ such that $E_{\lambda}^{n}(D)$ contains a disk of radius larger than $2 \pi$. It follows that there exists $z \in D$ with $\operatorname{Im} E_{\lambda}^{n}(z)=(2 k+1) \pi$ for some integer $k$. But then $E_{\lambda}^{n+1}(z) \in \mathbb{R}^{-}$, contradicting the fact that $\operatorname{Re} E_{\lambda}^{n+1}(z)>1$. This proves that the complement of the basin of $q$ is nowhere dense. It also shows that the family of iterates of $E$ is not a normal family at any point in the complement. This establishes the second part of the proposition.

Our goal for the remainder of this section is to prove that $J\left(E_{\lambda}\right)$ is a Cantor bouquet. We first describe the crown. Let $N \in \mathbb{Z}^{+}$. For each $k \in \mathbb{Z}$ with $|k| \leq N$ we construct a rectangle $B(k)$ whose boundaries are:

(1) $x=\log (1 / \lambda)$ on the left;

(2) $y=(2 k \pm 1) \pi$ above and below;

(3) $x=\nu$ on the right, where $\nu$ satisfies $\lambda e^{\nu}-\nu>(2 N+1) \pi$. 
Clearly, there exists $\nu_{0}$ such that, if $\nu>\nu_{0}$, (3) holds. See figure 2.

$$
\text { Let } \Lambda=\left\{z \mid E_{\lambda}^{i}(z) \in \bigcup_{k=-N}^{N} B(k) \text { for all } i \geq 0\right\} \text {. }
$$

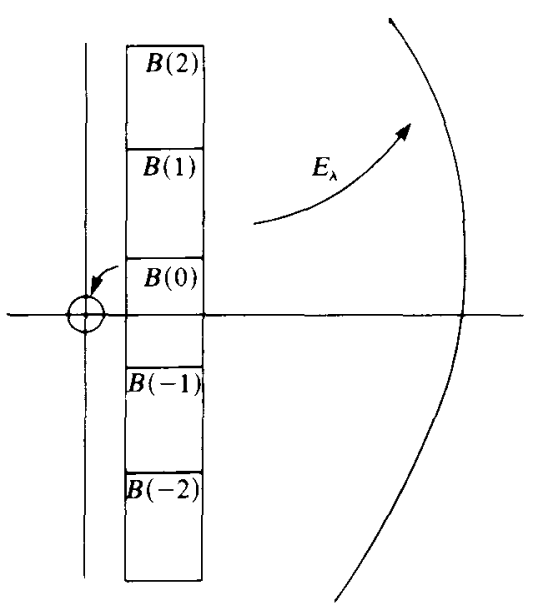

FIGURE 2

Proposition 2.2. (1) $\Lambda$ is homeomorphic to $\Sigma_{N}$.

(2) $E_{\lambda} \mid \Lambda$ is conjugate to the shift on $\Sigma_{N}$.

Proof. Each $B(k)$ is mapped by $\Sigma_{\lambda}$ onto the annulus $1 \leq r \leq \lambda e^{\nu}$ and therefore covers each other $B(j)$. Moreover, $\left|E_{\lambda}^{\prime}(z)\right|>1$ for all $z \in \bigcup_{k=-N}^{N} B(k)$. Therefore the preimage of $B(j)$ in $B(k)$ is a 'quadrilateral' completely contained in the interior of $B(k)$. Standard arguments similar to the Smale horseshoe construction [Sm] now complete the proof: a nested sequence of appropriate inverse images converges to the unique point $\Lambda$ with pre-assigned symbol sequence.

We now turn to the existence of the tails for points in $\Lambda$. Let $S_{\nu}$ denote the strip

$$
S_{\nu}=\{z|\operatorname{Re}(z) \geq \nu,| \operatorname{Im}(z) \mid \leq(2 N+1) \pi\} .
$$

We will show that if $\nu$ is large enough, then the set of points whose orbits remain for all time in $S_{v}$ is homeomorphic to $\Sigma_{N} \times[\nu, \infty)$.

LEMMA 2.3. There exists $\nu_{1}$ such that if $\nu \geq \nu_{1}$ and if both $z, E_{\lambda}(z) \in S_{\nu}$, then $\operatorname{Re}\left(E_{\lambda}(z)\right)>2 \operatorname{Re}(z)$.

The proof is a straightforward computation using the fact that vertical lines $x=\nu$ are mapped to circles $r=\lambda e^{\nu}$.

LeмmA 2.4. Choose $\nu_{2}>\nu_{0}$ such that $\lambda e^{\nu_{2}}>1$. Suppose $\nu \geq \nu_{2}$ and $E_{\lambda}^{j}(z) \in S_{\nu}$ for $j=0, \ldots, k$ then $\left|\left(E_{\lambda}^{k}\right)^{\prime}(z)\right| \geq \exp \nu\left(2^{k-1}-1\right)$. 
Proof. We have

$$
\begin{aligned}
\left|\left(E_{\lambda}^{k}\right)^{\prime}(z)\right| & =\left|\prod_{j=0}^{k-1} E_{\lambda}^{\prime}\left(E_{\lambda}^{j}(z)\right)\right| \\
& =\left|\prod_{j=0}^{k-1} E_{\lambda}^{j+1}(z)\right| \\
& \geq \lambda^{k} \prod_{j=0}^{k-1} \exp \left(2^{j} \operatorname{Re} z\right) \\
& \geq \lambda^{k} \exp \left(\nu 2^{k-1}\right) \exp \nu\left(2^{k-1}-1\right) \\
& \geq \exp \nu\left(2^{k-1}-1\right) .
\end{aligned}
$$

Let $t \in \mathbb{R}$ and define for any integer $k$ the square $\Delta(t+2 \pi i k)$ of sidelength $2 \pi$ centred at $t+2 \pi i k$. Note that the horizontal boundaries of $\Delta(t+2 \pi i k)$ lie along the lines $y=(2 k \pm 1) \pi$ and hence are mapped to $\mathbb{R}^{-}$by $E_{\lambda}$. We also define the substrip $S_{\nu}(j) \subset S_{\nu}$ to be

$$
\left\{z \in S_{\nu} \mid(2 j-1) \pi \leq \operatorname{Im}(z) \leq(2 j+1) \pi\right\} .
$$

LeMMA 2.5. (1) Suppose $\nu_{3}>\log \left((2 N+2) \pi e^{\pi} / \lambda\left(e^{\pi}-1\right)\right)$. If $t>\nu_{3}$, then the image of $\Delta(t+2 \pi i k)$ covers $\Delta\left(E_{\lambda}(t)+2 \pi i j\right)$ for any $j$ with $|j| \leq N$.

(2) Let $\nu>\nu_{3}+\pi$. Suppose $E_{\lambda}^{j}(t) \in S_{\nu}$ for $j=0,1, \ldots, k$. Let $s_{0}, s_{1}, \ldots, s_{k-1}$ be integers with $\left|s_{j}\right| \leq N$. Then there exists $z \in S_{\nu}$ with $E_{\lambda}^{j}(z) \in S_{\nu}\left(s_{j}\right)$ and $E_{\lambda}^{k}(z)=E_{\lambda}^{k}(t)$.

Proof. For the first part one checks easily that, if $t \geq \nu_{3}$, then

$$
\left|E_{\lambda}(t+\pi)\right|>E_{\lambda}(t)+\pi+(2 N+1) \pi .
$$

and

$$
\left|E_{\lambda}(t-\pi)\right|<E_{\lambda}(t)-\pi .
$$

For the second part, we use induction. First, recall that $t$ is real. If $k=1$, then $z=t+2 \pi i s_{0}$, so the result is true. If the result is true for $k-1$, then from part (1) we have $E_{\lambda}^{j}(z) \in \Delta\left(E_{\lambda}^{j}(t)+2 \pi i s_{j}\right)$ for $j=1, \ldots, k$. By (1), $E_{\lambda}$ maps $\Delta\left(t+2 \pi i s_{0}\right)$ over $\left(E_{\lambda}(t)+2 \pi i s_{1}\right)$. If $z \in S_{\nu}\left(s_{0}\right)$ and $\operatorname{Re}(z) \in[t-\pi, t+\pi]$, then $E_{\lambda}(z) \notin \Delta\left(E_{\lambda}(t)+2 \pi i s_{1}\right)$. This completes the proof.

Remark. The content of the second part of this lemma is that $E_{\lambda}$ behaves very much like $E_{\lambda} \mid \mathbb{R}$ on the set of points whose orbits remain for all time in $S_{\nu}$.

Let $\log$ be the branch of the natural logarithm taking values in $|\operatorname{Im}(z)|<\pi$. Let $L_{k}$ be the branch of the inverse of $E_{\lambda}$ which takes values in $S_{\nu}(k)$, i.e. $L_{k}(z)=$ $\log (z / \lambda)+2 \pi i k$. Let $s=\left(s_{0} s_{1} s_{2}, \ldots\right) \in \Sigma_{N}$. For each $k \geq 0$ we define

$$
\Phi_{k}(s, t)=L_{s_{0}} \circ \cdots \circ L_{s_{k}} \circ E_{\lambda}^{k+1}(t)
$$

for $t$ sufficiently large. Note that each $\Phi_{k}$ is well defined by lemma 2.5 .

Proposition 2.6. Let $\tau>v_{i}+\pi$ for $i=0,1,2,3$. Then, for each $s \in \Sigma_{N}, \Phi_{k}(s, t)$ converges uniformly on $[\tau, \infty)$ as $k \rightarrow \infty$. 
Proof. We have

$$
\begin{aligned}
& \left|\Phi_{k+1}(s, t)-\Phi_{k}(s, t)\right| \\
& \quad=\left|L_{s_{0}} \circ \cdots \circ L_{s_{k}}\left(E_{\lambda}^{k+1}(t)+2 \pi i s_{k}\right)-L_{s_{0}} \circ \cdots \circ L_{s_{k}}\left(E_{\lambda}^{k+1}(t)\right)\right|
\end{aligned}
$$

By lemma 2.4 we have

$$
\left|\Phi_{k+1}(s, t)-\Phi_{k}(s, t)\right| \leq e^{-\tau\left(2^{k}-1\right)}(2 N+1) \pi .
$$

This gives uniform convergence in $t$.

In particular, this proposition yields, for each $s \in \Sigma_{N}$, a continuous curve $\Phi(s, t)$ defined for $t \geq \tau$ in $S_{\tau}$. If $\mathbf{r} \in \Sigma_{N}$ with $\mathbf{r}=\left(s_{0} s_{1} \cdots s_{k} r_{k+1} r_{k+2} \cdots\right)$ then (*) implies that $\Phi(s, t)$ is close to $\Phi(\mathbf{r}, t)$, so that $\Phi$ is continuous in $s$. $\Phi$ is easily seen to be one-to-one as a map on $\Sigma_{N} \times[\tau, \infty)$. Let $\Lambda_{\tau}=\left\{z \in S_{\tau} \mid E_{\lambda}^{j}(z) \in S_{\tau}\right.$ for all $\left.j\right\}$. We claim that $\Omega: \Sigma_{N} \times[\tau, \infty) \rightarrow \Lambda_{\tau}$ is also surjective. For if $z \in \Lambda_{\tau}$, then there is a well-defined sequence $s$ associated to $z$ since the horizontal boundaries of $S_{\nu}\left(s_{i}\right)$ are mapped out of $S_{\nu}$ into $\mathbb{R}^{-}$by $E_{\lambda}$. Furthermore, $\left|\left(E_{\lambda}^{j}\right)^{\prime}(z)\right| \rightarrow \infty$ as $j \rightarrow \infty$. Thus, any small disk $U$ about $z$ is eventually expanded so that the diameter of its image exceeds $2 \pi$. It follows that there is a sequence of points $z_{k}$ in $U$ which converge to $z$ and which satisfy $E_{\lambda}^{j}\left(z_{k}\right) \in S_{\nu}\left(s_{j}\right)$ for $j<k$ but $E_{\lambda}^{k}\left(z_{k}\right) \in \mathbb{R}$. Let $t^{*}$ denote the supremum of the real parts of points in $U$. Then it follows immediately that

$$
E_{\lambda}^{k}\left(t^{*}\right) \geq E_{\lambda}^{k}\left(z_{k}\right) \text {. }
$$

Hence $z_{k}=\Phi_{k}\left(\mathbf{r}_{k}, t_{k}\right)$ for some $\mathbf{r}_{k} \in \Sigma_{N}$ and $t_{k} \leq t^{*}$. It follows that the sequence $\Phi_{k}\left(\mathbf{r}_{k}, t_{k}\right)$ converges to $z$. Thus, we have proved:

Proposition 2.7. $\Lambda_{\tau}$ is homeomorphic to $\Sigma_{N} \times[0, \infty)$.

Let $z$ be any point whose orbit lies for all time in $S_{\log 1 / \lambda}$ and which does not lie in $\Lambda$, i.e. $z$ does not lie in the crown of the $N$-bouquet. It follows easily that there exists an $m$ for which $E_{\lambda}^{m}(z) \in \Lambda_{r}$. Hence we may pull back the curve in $\Lambda_{\tau}$ through any such point. It follows that $\left\{z \mid E_{\lambda}^{j}(z) \in S_{\log 1 / \lambda}\right.$ for all $\left.j\right\}$ is an $N$-bouquet. See figure 3 .

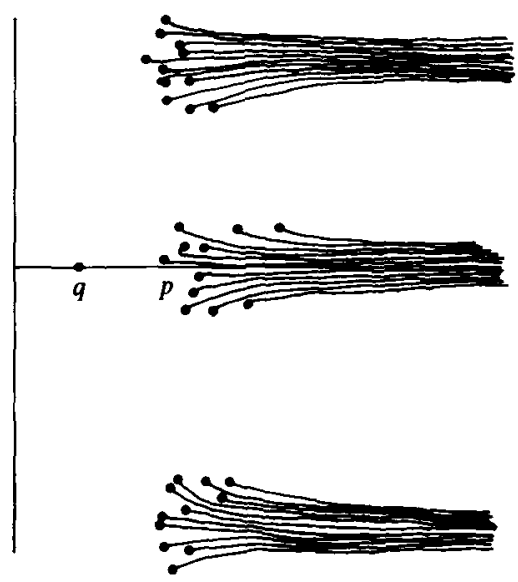

Figure 3 
Remarks. (1) All of the tails are asymptotic at infinity in the following sense. Let $\Phi(t)$ be the parametrization of any tail. Define the $\delta$-angle about $\Phi$ to be $B_{\delta t}(\Phi(t))$. If $\Phi_{1}(t)$ is any other tail, then there exists $\tau$ such that for $t>\tau, \Phi_{1}(t)$ belongs to the $\delta$-angle about $\Phi$.

(2) The vertical boundary $x=\log (1 / \lambda)$ of the exponential tract also has dynamical significance. Recall that a Julia ray is a ray such that in any $\delta$-angle about it, the conclusion of the Great Picard theorem holds. The line $x=\log (1 / \lambda)$ is a Julia ray in the direction of both increasing and decreasing imaginary part.

(3) If $\lambda>1 / e$, the above ideas must be modified somewhat. First, it is known that $J\left(E_{\lambda}\right)=C$ in this case [BR], [D], [Mi]. So $J(E \lambda)$ is certainly not a Cantor bouquet. Moreover, any vertical line $x=c$ is mapped to a circle with radius greater than $c$, so the above construction fails. Nevertheless, if we eliminate certain of the strips $S_{\tau}(k)$ for $|k|<K$, then the above construction does work and there is an invariant subset of $J\left(E_{\lambda}\right)$ which is a Cantor bouquet.

(4) One can use this construction to show that the Lebesgue measure of $J\left(E_{\lambda}\right)$ is zero. This is a recent result of Eremenko and Ljubic [EL1]. McMullen has recently shown [Mc] that $J\left(E_{\lambda}\right)$ has Hausdorff dimension 2.

\section{Hyperbolic exponential tracts}

In this section we give specific conditions that guarantee that the set of points whose orbits remain inside a given exponential tract $T$ is a Cantor bouquet. We assume throughout that $T$ is contained in a sector $S$ and that the entire, transcendental map $E$ is critically finite. Let $C_{r}$ denote the circle given by $|z|=r$ and $B_{r}$ the closed disk, $|z| \leq r$. We assume that all of the critical and asymptotic values of $E$ lie in the interior of $B_{r}$. We also assume that there exists $\rho$ such that $E \mid T$ covers the complement of the disk $B_{\rho}$ and that $T \cap B_{\rho}=\varnothing$. We will discuss later the changes that ensue if $T$ and $B_{\rho}$ overlap.

Since $T \subset S$, we may fix a ray $\zeta=\zeta(r)=r e^{i \hat{\theta}}$ which is disjoint from $S$ and defined for $r \geq \rho$, and use the pre-images of this ray in $T$ to set up the fundamental domains for the Cantor bouquet. More precisely, let $\gamma_{i}=\gamma_{i}(r)$ for $i \in \mathbb{Z}$ denote the pre-images of $\zeta$ in $T$. That is, $\gamma_{i}(r)=E^{-1}(\zeta(r))$ for an appropriate branch of $E^{-1}$. We may choose the index $i$ in the natural way so that $\gamma_{i}$ and $\gamma_{i+1}$ are adjacent for each $i$. Clearly, $\gamma_{i}(r)$ tends to $\infty$ as $r \rightarrow \infty$.

The curves $\gamma_{i}$ and $\gamma_{i+1}$ bound a strip which serves as a fundamental domain for $E \mid T$. See figure 4 . Let us denote this strip by $T_{i}$. Let $W_{N}=\bigcup_{i=-N}^{N} T_{i}$. Our goal will be to give conditions that guarantee that

$$
\Lambda_{N}=\left\{z \in W_{N} \mid E^{j}(z) \in W_{N} \text { for all } j \geq 0\right\}
$$

is homeomorphic to a Cantor $N$-bouquet. Basically, the conditions which we will give amount to an assumption on the direction in which points approach $\infty$ as well as the rate at which they approach $\infty$ under iteration. As such, these conditions are reminiscent of the conditions which define a hyperbolic fixed point for a map. Indeed, if we regard $\infty$ as a fixed point for $E \mid T$ (and not globally), then this is a fairly good analogy.

Our first assumption is that all of the fundamental domains approach infinity in the same direction. 


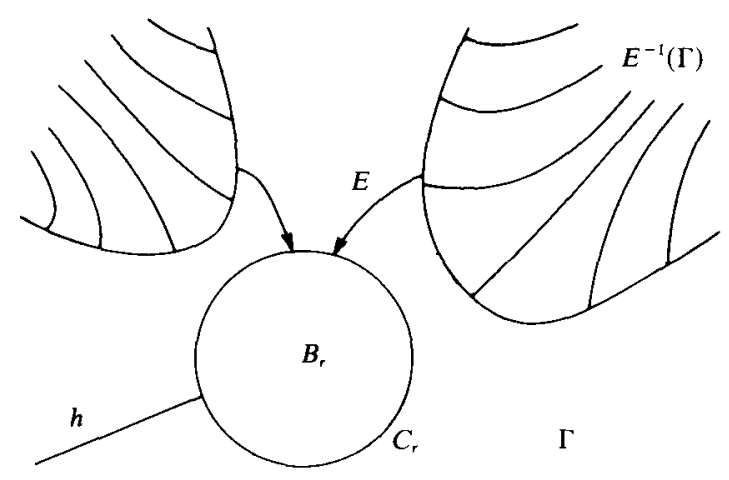

FIGURE 4. Fundamental domains in exponential tracts.

Definition 3.1. $E \mid T$ has asymptotic direction $\theta^{*}$ if $\gamma_{i}(r)$ is $C^{1}$-asymptotic to a straight line with direction $\theta^{*}$ for each curve $\gamma_{i}$ defining the fundamental domains.

Note that, if $E \mid T$ has asymptotic direction $\theta^{*}$, then all of the $\gamma_{i}$ which lie in $W_{N}$ meet circles of sufficiently large radius in a unique point. That is, there exists $R_{0}$ such that, if $r>R_{0}$ and $\gamma_{i} \subset W_{N}$, then the equation $\left|\gamma_{i}\right|=r$ has a unique solution. As a consequence, we may assume that the tails of each of the $\gamma_{i}$ in $W_{N}$ are parametrized by $r$, at least for $r \geq R_{0}$.

Besides assuming that the $\gamma_{i}$ tend to $\infty$ in a certain direction, we also assume that points approach $\infty$ with a certain speed under iteration. Our second assumption is:

Definition 3.2. $T$ is a hyperbolic exponential tract if there exist positive constants $R_{1}, \alpha, C$ such that, if $z$ and $E(z)$ lie in $W_{N}$, with $|z|=r \geq R_{1}$, then

(i) $|E(z)|>C e^{r^{\alpha}}$

(ii) $\left|E^{\prime}(z)\right|>C e^{r^{\alpha}}$

(iii) $\left|\arg \left(E^{\prime}(z)\right)\right|<C e^{-r^{\alpha}}$

We remark that $R_{1}, \alpha$, and $C$ may depend on $N$. Our aim is to prove the following theorem.

THEOREM 3.3. Let $E$ be a critically finite, entire, transcendental map. Let $T$ be a hyperbolic exponential tract on which $E$ has asymptotic direction $\theta^{*}$. Then, for each $N, \Lambda_{N}$ is a Cantor $N$-bouquet. Consequently,

$$
J_{T}(E)=\left\{z \mid E^{j}(z) \in T \text { for all } j \geq 0\right\}
$$

contains a Cantor bouquet.

To prove this theorem, we need several lemmas. Let $g(r)=C e^{r^{\alpha}}$ for $r \geq R_{1}$ where $R_{1}, C, \alpha$ are as given in definition 3.2. The proof of the following lemma is straightforward.

LEMmA 3.4. (1) There exists $R_{2} \geq R_{1}$ such that, if $r>R$, then $g(r)>r$. Moreover, $g^{n}(r) \rightarrow \infty$ if $r>R_{2}$.

(2) Let $\varepsilon>0$. Then there exists $R_{3}=R_{3}(\varepsilon)$ such that, if $r>R_{3}$, then

$$
\sum_{k=1}^{\infty} 1 / g^{k}(r)<\varepsilon
$$


For simplicity of notation, let us denote the set of points $\left\{z \in T_{i}|| z \mid \geq r\right\}$ by $T_{i}(r)$. We will also concentrate for the moment on a single fundamental domain $T_{0}$, later extending these results to the collection of domains $W_{N}$.

LEMMA 3.5. Let $T$ be a hyperbolic exponential tract for $E$ with asymptotic direction $\theta^{*}$. Then $E$ has a unique fixed point in $T_{0}$, and this fixed point is repelling.

Proof. By lemma 3.4, we may choose $R_{2}$ so that $C e^{r^{\alpha}}>r$ provided $r \geq R_{2}$. Then we choose $R \geq \max \left(R_{0}, R_{2}\right)$ where $R_{0}$ is such that the boundaries $\gamma_{0}$ and $\gamma_{1}$ are functions of $r$ for $r \geq R_{0}$, as described after definition 3.1.

Consider the subset $U_{R}=T_{i}-T_{i}(R)$. That is, $U_{R}=\left\{z \in T_{i}|| z \mid \leq R\right\}$. The closure $\bar{U}_{R}$ is easily seen to be homeomorphic to a rectangle, two of whose boundary components lie in $\gamma_{0}$ and $\gamma_{1}$. The third lies in $\partial T$, and the fourth lies in $C_{R} . E$ expands $\bar{U}_{R}$ onto an annular region bounded by $C_{\rho}$ and the image of $C_{R} \cap T_{0}$. By condition (i) in definition 3.2, this latter image lies in the region $|z|>R$, while the former image lies in the exterior of $T$. Since $E \mid \bar{U}_{R}$ is one to one except on $\gamma_{0} \cup \gamma_{1}$, it follows that $E$ has a fixed point in $U_{R}$. We henceforth denote this fixed point by $z_{0}$. Note that $z_{0}$ is the unique point whose orbit remains for all time in $\bar{U}_{R}$.

Now let us consider the set of points $\Lambda_{0}$ which remain for all time in the larger set, $T_{0}$. We remark that $\Lambda_{0}$ is a closed set, since

$$
\Lambda_{0}=\bigcap_{n=0}^{\infty} \phi^{-n}\left(T_{0}\right)
$$

where $\phi$ is the branch of the inverse of $E$ taking values in $T_{0}$. Moreover, provided $|E(z)|>C e^{r^{\alpha}}$ as in assumption (i) in definition 3.2, it follows easily that there is exactly one fixed point in $\Lambda_{0}$ and all other points in $\Lambda_{0}$ tend to $\infty$ under iteration of $E$. Hence, $\Lambda_{0}$ 'connects' the fixed point to $\infty$. To prove that $\Lambda_{0}$ is actually a curve, however, necessitates the full power of the definition of hyperbolicity.

In analogy with our construction for the exponential map, we will first work with those points which remain in $T_{0}$ and have sufficiently large modulus. Let

$$
\Lambda_{0}(R)=\left\{z \in T_{0}(R) \mid E^{j}(z) \in T_{0}(R) \text { for all } j \geq 0\right\} .
$$

LEMMA 3.6. If $R$ is sufficiently large, then $\Lambda_{0}(R)$ is a continuous curve.

Proof. We will construct a pair of sequences of $C^{\infty}$ curves $\alpha_{k}(r)$ and $\beta_{k}(r)$ which will be defined for $r \geq R$ and have the following properties:

(1) $\left|\alpha_{k}(r)\right|=\left|\beta_{k}(r)\right|=r$;

(2) $\Lambda_{0}(R)$ lies in the strip contained in $T_{0}(R)$ and bounded by $\alpha_{k}$ and $\beta_{k}$ for each $k$;

(3) $\left|\alpha_{k}(r)-\beta_{k}(r)\right| \rightarrow 0$ uniformly as $k \rightarrow \infty$.

As a consequence, $\Lambda_{0}(R)$ is a continuous curve which may be parametrized by $r \geq R$.

To define $R$ and the $\alpha_{k}$ and $\beta_{k}$, we first recall that there exists $R_{0}$ such that, if $r \geq R_{0}$, then both $\gamma_{0}$ and $\gamma_{1}$ meet $C_{r}$ in a unique point. Here, $\gamma_{0}$ and $\gamma_{1}$ are the boundaries of $T_{0}$. Hence we may parametrize both of these curves by $r \geq R_{0}$. We set $\alpha_{0}=\gamma_{0}$ and $\beta_{0}=\gamma_{1}$. Since both $\alpha_{0}$ and $\beta_{0}$ have asymptotic direction $\theta^{*}$, we may 
further assume that, if $r \geq R_{0}$, then

$$
\begin{aligned}
& \left|\arg \alpha_{0}^{\prime}(r)-\theta^{*}\right|<\varepsilon / 2, \\
& \left|\arg \beta_{0}^{\prime}(r)-\theta^{*}\right|<\varepsilon / 2
\end{aligned}
$$

Let

$$
\delta_{0}=\sup _{r \geq R}\left|\alpha_{0}(r)-\beta_{0}(r)\right| .
$$

Since both $\alpha_{0}$ and $\beta_{0}$ are asymptotic to lines with the same direction, it follows that $\delta_{0}<\infty$.

Now let $z \in \Lambda_{0}(R)$ and suppose that $|z|=r$. If we choose $R>R_{1}$, then we have $\left|E^{k}(z)\right|>g^{k}(r)$ for all $k \geq 0$, where $g(r)=C e^{r^{\alpha}}$. If $R>R_{2}$, then lemma 3.4 shows that $E^{k}(z) \rightarrow \infty$ as $k \rightarrow \infty$.

If $R>R_{1}$, then we also have that

$$
\left|\arg \left(E^{\prime}(z)\right)\right|<\frac{1}{g(r)} .
$$

By the chain rule, we have

$$
\begin{aligned}
\left|\arg \left(E^{k}\right)^{\prime}(z)\right| & =\left|\arg \prod_{i=0}^{k-1} E^{\prime}\left(E^{i}(z)\right)\right| \\
& \leq \sum_{i=0}^{k-1}\left|\arg E^{\prime}\left(E^{i}(z)\right)\right| \\
& \leq \sum_{i=0}^{k-1} \frac{1}{g^{i}(r)}
\end{aligned}
$$

If we choose $R>R_{3}$, then lemma 3.4 guarantees that

$$
\left|\arg \left(E^{k}\right)^{\prime}(z)\right|<\varepsilon / 2 \text {. }
$$

for all $k$

Now let $\phi$ denote the branch of the inverse of $E \mid T$ which takes values in $T_{0}$. We define the curves $\alpha_{k}$ and $\beta_{k}$ by $\phi^{-k}\left(\alpha_{0}\right)$ and $\phi^{-k}\left(\beta_{0}\right)$. Provided $R$ is chosen large enough, $(*)$ guarantees that $\alpha_{k}$ and $\beta_{k}$ have arguments which differ from $\theta^{*}$ by no more than $\varepsilon / 2$. Hence $\alpha_{k}$ and $\beta_{k}$ may also be parametrized by $r$. That is, $\alpha_{k}(r)$ is the unique point in $C_{r} \cap T_{0}$ whose orbit stays in $T_{0}$ under $E, E^{2}, \ldots, E^{k}$ and which satisfies $E^{k}\left(\alpha_{k}(r)\right) \in \alpha_{0}$.

We thus assume that $R \geq \max \left(R_{i}\right)$. Let $\delta_{k}$ be the $C^{0}$-distance between $\alpha_{k}$ and $\beta_{k}$ for $r \geq R$ i.e.,

$$
\delta_{k}=\sup _{r \geq R}\left(\left|\alpha_{k}(r)-\beta_{k}(r)\right|\right)
$$

Using conditions (ii) and (iii) in definition 3.2, it follows immediately that there is a constant $\nu<1$ such that, if $r \geq R$, then $\delta_{k}<\nu^{k} \delta_{0}$. Consequently, $\delta_{k} \rightarrow 0$ uniformly for $R$ sufficiently large. Thus the $\alpha_{k}$ and $\beta_{k}$ converge uniformly to $\Delta_{0}(R)$.

This completes the proof of lemma 3.6.

This shows that $\Lambda_{0}(R)$ is a continuous curve parametrized by $r$ for $r \geq R$. To show that $\Lambda_{0}$ is homeomorphic to $[0, \infty)$, we use a fundamental domain argument. Let $D$ be the complement of $T_{0}(R)$ in the entire strip $T_{0} . D$ is homeomorphic to a disk 
and is mapped inside itself by the inverse map $\phi$. Moreover, by the Schwarz lemma, any point $z \in D$ tends to $z_{0}$ under iteration of $\phi$. Now the region $F=D-\phi(D)$ forms a fundamental domain for $\phi$. Note that $\Lambda_{0}(R)$ meets this region in a unique point. Denote this point by $p_{0}$. Then $\phi\left(\Lambda_{0}(R)\right)$ meets $F$ in a continuous curve which extends from $p_{1}=\phi\left(p_{0}\right)$ to $p_{0}$ in $F$. Hence we may parametrize this portion of $\Lambda_{0}$ by any continuous map defined on the interval $(R / 2, R]$ and taking $R / 2$ to $p_{1}$. Taking the $j$ th image of this curve and then choosing any parametrization defined on intervals of the form $\left(R / 2^{j+1}, R / 2^{j}\right]$ (with endpoints matching appropriately) shows that $\Lambda_{0}-z_{0}$ may be continuously parametrized on the interval $(0, \infty)$. Since $F$ is a fundamental domain, we may extend this parametrization continuously to 0 , with 0 corresponding to $z_{0}$.

This proves theorem 3.3 in the case of a single fundamental domain. The case of $W_{N}$ is essentially the same, provided $R$ is chosen large enough so that each of the fundamental rectangles are expanded over all of the rectangles. This produces the shift map on the invariant Cantor set, much as in the case of the exponential map. More precisely, as above, we may choose $R$ large enough so that $U_{N}=$ $\left\{z \in W_{N}|| z \mid \leq R\right\}$ is a union of 'rectangles', $D_{-N}, \ldots, D_{N}$, with $D_{j} \subset T_{j}$. Choosing $R$ larger if necessary, we may guarantee that $E$ is one-to-one on the interior of each $D_{j}$ and that $E \mid D_{j}$ covers $U_{N}$. Hence, for each $j$ with $-N \leq j \leq N$, we may define the branch of the inverse of $E$ given by $\phi_{j}: U_{N} \rightarrow D_{j}$. In the Poincaré metric on $U_{N}$, each $\phi_{j}$ is a strict contraction. That is, there exists $\mu<1$ such that $\left|\phi_{j}(z)-\phi_{j}(w)\right|<$ $\mu|z-w|$. Now let $(s)=\left(s_{0}, s_{1}, s_{2}, \ldots\right)$ be any sequence in $\Sigma_{N}$. Let $\Psi_{k}=\phi_{s_{0}} \circ \ldots \circ \phi_{s_{k}}$. By the above considerations, the $\Psi_{k}$ converge uniformly in the Poincaré metric to a constant map $z \mapsto z_{s}$. The point $z_{s}$ is clearly unique and has itinerary $s$. The collection of all such $z_{s}$ for $s \in \Sigma_{N}$ gives an invariant Cantor set on which the map $E$ is topologically conjugate to the shift map, just as in the case of the exponential map. The construction of the tails associated to each $z_{s}$ proceeds as above. We therefore leave the details to the reader. This completes the proof of theorem 3.3.

Remark. Our proof of the existence of the tails in the $N$-bouquet is somewhat different from the proof we gave in the special case of the exponential map in $\S 2$. This proof can be adapted to the general case provided we have the existence of at least one tail in the bouquet already. Symmetry conditions often give the existence of this tail without difficulty, as we shall show in the next section.

\section{Applications and extensions}

In this section we gather together several applications of the previous results and show how to eliminate some of the restrictions imposed in $\S 3$. First consider the map $S_{\lambda}(z)=\lambda \sin z$. If $\lambda$ is real with $0<\lambda<1$, then $S_{\lambda}$ has an attracting fixed point at 0 . Moreover, since $S_{\lambda}(i y)=i \lambda \sinh (y), S_{\lambda}$ preserves the imaginary axis. There are two other fixed points on this axis, located at $i p_{ \pm}(\lambda)$, where $p_{+}>0$ and $p_{-}=-p_{+}$. These fixed points are repelling, and the portion of the positive imaginary axis connecting $i p_{+}(\lambda)$ to $\infty$ consists of points which tend to $\infty$. We will see below that this is the tail attached to $i p_{+}(\lambda)$ in $J\left(S_{\lambda}\right)$. Similarly, $i p_{-}(\lambda)$ has a tail on the negative imaginary axis. 
We claim that $J\left(S_{\lambda}\right)$ contains a pair of Cantor bouquets, one tending to $\infty$ in the direction of the positive imaginary axis and one in the direction of the negative imaginary axis. To see this, we note that $S_{\lambda}$ is critically finite: there are only two critical values at $\pm \lambda$ and no (finite) asymptotic values. Choose $\nu$ such that $0<\nu<$ $p_{+}(\lambda)$. The horizontal line $y=\nu$ is mapped by $S_{\lambda}$ onto an ellipse with centre at 0 . This ellipse meets the imaginary axis at $i \lambda \sinh (\nu)<\nu$ since $\nu<p_{+}(\lambda)$. Moreover, $S_{\lambda}$ maps the half plane $H_{+}$given by $\operatorname{Im}(z)>\nu$ onto the exterior of this ellipse. By the results of $\S 1$, this region is an exponential tract for $S_{\lambda}$. We may choose the negative imaginary axis to define the fundamental domains in $H_{+}$. Since the vertical half-lines given by $x=(2 k+1) \pi, y \geq \nu$ for $k \in \mathbb{Z}$ are mapped by $S_{\lambda}$ onto this axis, it follows that $H_{+}$has asymptotic direction $\theta_{*}=\pi / 2$. Furthermore, since $S_{\lambda}(z)=$ $(\lambda / 2 i)\left(e^{i z}-e^{-i z}\right)$, one may check readily that $H_{+}$is a hyperbolic exponential tract.

Similar results hold as well for $H_{-}=\{z \mid \operatorname{Im}(z)<-\nu\}$. As a consequence, we have:

THEOREM 4.1. $J\left(S_{\lambda}\right)$ contains a pair of Cantor bouquets for $0<\lambda<1$, one in $H_{+}$and one in $H_{-}$.

One has a similar theorem for $C_{\lambda}(z)=\lambda \cos z$ provided $\lambda \in \mathbb{R}$ is chosen so that $C_{\lambda}$ has a fixed sink. This is true for $|\lambda|<1$, as one may easily check.

Theorem 3.3 yields an interesting alternative definition of the Julia set of an entire function which meets our growth and asymptotic direction requirements in all exponential tracts.

THEOREM 4.2. Suppose $E$ is an entire transcendental function which is critically finite and which has an exponential tract $D$ which is hyperbolic with a given asymptotic direction. Then

$$
J(E)=\text { closure }\left\{z \mid E^{j}(z) \rightarrow \infty\right\} .
$$

Proof. By a result of Fatou $[\mathbf{F}], J(E)$ is the closure of the set of pre-images of any point in $J(E)$. Hence we simply take this point to be a point which lies on one of the tails in $J_{D}(E)$.

Remarks. (1) Note how this differentiates the entire, transcendental case from the case of polynomial maps: for polynomials, points which tend to $\infty$ under iteration are never in the Julia set.

(2) For entire maps which have a hyperbolic exponential tract with an asymptotic direction, there are thus two seemingly contradictory definitions of the Julia set. One gives $J(E)$ as the closure of the immediate unbounded orbits, while the other gives $J(E)$ as the closure of perhaps the simplest bounded orbits, the periodic orbits (which are repelling). Of course, our definition of Cantor bouquet explains this apparent contradiction.

(3) This definition of $J(E)$ yields an easy algorithm to compute the Julia set numerically: one simply computes which points tend to go far from 0 in the required asymptotic directions.

(4) Let $T$ be a hyperbolic exponential tract. Since $E \mid T$ is a universal covering map, the Julia set $J_{T}(E)$ has infinitely many symmetries given by the various deck 
transformations on $T$. This accounts for some of the self-similarity observed in computer pictures of Julia sets of entire functions.

Our final topic in this section deals with the behaviour of the Cantor bouquets in case an exponential tract overlaps the disk which contains all of the critical and asymptotic values. In this case there may occur bifurcations or other changes in the structure of the Julia set.

One such bifurcation involves the exchange of 'hairs' among the various points in the crown of the bouquet. For example, consider the family $S_{\lambda}(z)=\lambda \sin (z)$ for $\lambda \in \mathbb{R}$. For $0<\lambda<1$, the Julia set contains two distinct Cantor bouquets, one in the upper half-plane $H_{+}$given by $\operatorname{Im}(z) \geq \nu$, and the other in the symmetrically located lower half-plane, $H_{-}$. The two repelling fixed points at $i p_{ \pm}(\lambda)$ have tails or hairs attached as described above. As $\lambda$ increases through 1, a bifurcation occurs: 0 ceases to be an attracting fixed point and two new attractors appear at $\pm q(\lambda)$ on the real line. One may check as before that $S_{\lambda}$ still preserves the imaginary axis, but this time all points except 0 tend to infinity under iteration. Thus the hairs in the Cantor bouquet previously attached to $i p_{ \pm}(\lambda)$ are now permanently attached to 0 for $\lambda>1$. For more details, see [D3]. See figure 5.

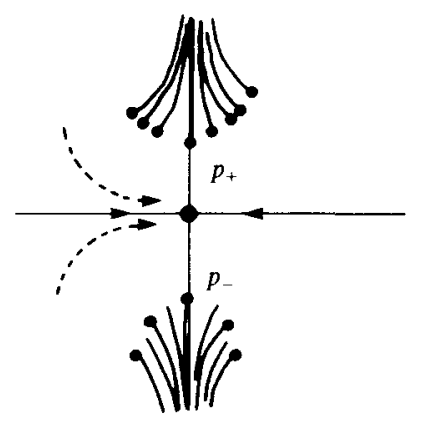

$\lambda<1$



$\lambda>1$

FIGURE 5. The exchange of hairs of $S_{\lambda}$ at $\lambda=1$.

We remark that similar exchanges simultaneously occur at $\lambda=1$ along all of the pre-images of the imaginary axis.

There is a second, completely different type of bifurcation which may occur when the exponential tract overlaps the disk containing the critical values. This is an explosion of the Julia set. For example, consider the family $E_{\lambda}(z)=\lambda \exp (z)$. For $0<\lambda<1 / e$, we have shown that $J\left(E_{\lambda}\right)$ is a single Cantor bouquet. For $0<\lambda<1 / e$, the asymptotic value at 0 tends to the single attracting fixed point on the real axis. But when $\lambda>1 / e, E_{\lambda}^{n}(0) \rightarrow \infty$. By Sullivan's theorem [S] as extended to entire maps by Goldberg and Keen [GK], the Julia set must be all of $\mathbb{C}$ in this case. This example shows that bifurcations also occur when the critical and asymptotic values fall onto one of the hairs in the Cantor bouquet. For more details, see [D1]. 


\section{REFERENCES}

[Ba1] I. N. Baker. Repulsive fixpoints of entire functions. Math. Z. 104 (1968), 252-256.

[Ba2] I. N. Baker. An entire function which has wandering domains. J. Austral. Math. Soc. 22 (1976), 173-176.

[Ba3] I. N. Baker. Wandering domains in the iteration of entire functions. Proc. London Math. Soc. 49 (1984), 563-576.

[BR] I. N. Baker \& P. J. Rippon. Iteration of exponential functions. Ann. Acad. Sci. Fenn. Ser. I A Math 9 (1984), 49-77.

[B] P. Blanchard. Complex analytic dynamics on the Riemann sphere. Bull. Amer. Math. Soc. 11 (1984), 85-141.

[D1] R. Devaney. Bursts into chaos. Phys. Lett. 104 (1984), 385-387.

[D2] R. Devaney. Structural instability of Exp (z). Proc. Amer. Math. Soc. 94 (1985), 545-548.

[D3] R. Devaney. Exploding Julia sets. To appear in Proc. Conf. on Chaotic Dynamics, Georgia Tech., 1985.

[D4] R. Devaney. Julia sets and bifurcation diagrams for exponential maps. Bull. Amer. Math. Soc. 11 (1984), 167-171.

[DH1] A. Douady \& J. Hubbard. Itération des polynomes quadratiques complexes. CRAS 294 (Janvier 1982).

[DH2] A. Douady \& J. Hubbard. Etude dynamique des polynomes complexes. Publ. Math. D'Orsay (preprint).

[DK] R. Devaney \& M. Krych. Dynamics of Exp (z). Ergod. Th. \& Dynam. Sys. 4 (1984), 35-52.

[EL1] A. Eremenko \& M. Ljubic. Iterates of entire functions. Preprint. UkrSSR Acad. Sci. Kharkov. (1984), No. 6.

[EL2] A. Eremenko \& M. Ljubic. Structural stability in some entire functions. Preprint. UkrSSR Acad. Sci. Kharkov. (1984), No. 29.

[F] P. Fatou. Sur l'itération des fonctions transcendantes entières. Acta Math. 47 (1926), 337-370.

[GK] L. Goldberg \& L. Keen. A finiteness theorem for a dynamical class of entire functions. To appear.

[Ma] B. Mandelbrot. The Fractal Geometry of Nature. Freeman, 1982.

[Mc] C. McMullen. Area and Hausdorff dimension of Julia sets of entire functions. Preprint.

[Mi] M. Misiurewicz. On iterates of $e^{z}$. Ergod. Th. \& Dynam. Sys. 1 (1981), 103-106.

[MSS] R. Mañe, P. Sad \& D. Sullivan. On the dynamics of rational maps. To appear.

[Sm] S. Smale. Diffeomorphisms with infinitely many periodic points. In Differential and Combinatorial Topology, Princeton University Press. Princeton, N.J. (1965), 63-80.

[S] D. Sullivan. Quasi-conformal homeomorphisms and dynamics I and III. Preprints. 